\title{
Myiasis Associated with Oral Squamous Cell Carcinoma - A Literature Review
}

\author{
Sadeq Ali Al-Maweri ${ }^{1 *}$, Ghadah AAl-Sufyani ${ }^{2}$, Bassel Tarakji ${ }^{3}$,Saleem Abdulrab ${ }^{4}$
}

\begin{abstract}
Advanced oral squamous cell carcinoma (SCC) is a possible risk factor for myiasis, a parasitic infestation of vital tissue of humans or other mammals by dipterous larvae (maggots). Oral myiasis is a rare entity, and is mostly associated with various medical and anatomical conditions, such as neglected mandibular fracture, lip incompetence, cerebral palsy, poor oral hygiene, suppurative lesions, and cancerous wounds. Larvae cause itching and irritation due to their crawling movements and can destroy vital tissues, inducing serious or even life-threating hemorrhage. The aim of the present article was to highlight the occurrence of oral myiasis in association with squamous cell carcinoma and also to highlight the treatment and preventive approaches for such cases. A literature search was performed using MEDLINE for articles published in English relating to the occurrence of oral myiasis in oral SCC. Our search revealed 6 reports on myiasis associated with oral SCC. The surgical debridement of infected tissue with the removal of maggots is the treatment of choice in most cases of oral myiasis.
\end{abstract}

Keywords: Myiasis - association - oral squamous cell carcinoma

Asian Pac J Cancer Prev, 16 (12), 4997-4999

\section{Introduction}

Squamous cell carcinoma (SCC) is the most common type of oral cancer, which forms more than $90 \%$ of all oral malignancies (Ferlay et al, 2010; Krishna Rao et al., 2013). Advanced oral cancers can cause significant morbidity and mortality. They infiltrate and destroy tissues thereby causing disfigurement, loss of function, pain, bleeding and necrosis (Ferlay et al., 2010). A rare complication of advanced SCC is myiasis (Gopalakrishnan et al., 2008; Wollina et al., 2014).

Myiasis is a term derived from the Greek word "myia,"meaning invasion of vital tissues of humans or other mammals by fly larvae (Pereira et al., 2010).The term "myiasis" was coined by F.W. Hope in 1840 to refer to diseases of human originating specifically with dipterous larvae, as opposed to those caused by insect larvae in general, scholechiasis (Bhagawati et al., 2013).

German entomologist Fritz Zumpt defined myiasis as "the infestation of live human and vertebrate animals with dipterous larvae, which at least for a period, feed on the host's dead or living tissue, liquid body substances, or ingested food" (Zumpt, 1965). Myiasis can be seen worldwide, but its incidence is higher in tropical and subtropical regions of Africa and America due to the favorable climatic conditions of heat and humidity (Saravanan et al., 2015)
Clinically, myiasia is classified as either primary or secondary. Primary myiasis is a rare in humans and is caused by biophagous larvae that feed on living tissue. Secondary myiasis is caused by the necrobiophagous flies that feed on necrotic tissue. This is the more common type and attacks patients with necrotic cavity lesions (Antunes et al., 2011).

Human myiasis is found among the elderly and abandoned individuals, as well as in interns of geriatric hospitals and mental institutions presenting poor hygienic habits (Gabriel et al., 2008).

In humans, the sites most commonly affected are skin, nose, ears, eyes, anus, vagina, and oral cavity (Saravanan et al., 2015). Oral myiasis, first described by Laurence in 1909, is a rare entity, and is mostly associated with medical and anatomical conditions, such as neglected mandibular fracture, lip incompetence, cerebral palsy, mouth breathing, anterior open bite, dental extractions, patients undergoing mechanical ventilation, and cancrum oris (Antunes et al., 2011). Other predisposing factors are anterior open bite, severe halitosis, poor oral hygiene, ulcerative lesions and oral malignancies (Sankari and Ramakrishnan, 2010; Bhagawati et al., 2013; Aggrawal et al., 2014). Myiasis is a possible complication of cancerous lesions. It has been seen more often in association with cutaneous cancer (Wollina et al., 2014). Although a rare, many oral myiasis cases associated with squamous cell

${ }^{\prime}$ Department of Oral and Maxillofacial Sciences, Al-Farabi Colleges of Dentistry and Nursing, Saudi Arabia; Department of Oral Medicine and Diagnosis, Sana'a University, ${ }^{2}$ Department of Dental Surgery, Al-Kuwait Teaching Hospital, Sana'a Yemen, ${ }^{3}$ Department of Oral and Maxillofacial Sciences, Al-Farabi Colleges of Dentistry and Nursing ${ }^{4}$ Department of Restorative Dental Sciences, Alfarabi Colleges, Riyadh, Saudi Arabia*For correspondence: sadali05@hotmail.com 


\begin{tabular}{|c|c|c|c|c|c|c|}
\hline No & Author & Year & Country & $\begin{array}{l}\text { No of } \\
\text { cases }\end{array}$ & Localization & Therapy \\
\hline 1 & Gabriel et al. & 2008 & Brazil & 1 & Floor of the mouth & Mechanical removal \\
\hline 2 & Carvalho et al. & 2008 & Brazil & 1 & Buccal mucosa & Mechanical removal, antiseptics \\
\hline 3 & Gopalakrishnan et al. & 2008 & India & 2 & Buccal mucosa; lower lip & Not mentioned \\
\hline 4 & Pessoa \& Galvão & 2011 & Brazil & 1 & Mandible & Mechanical removal, antiseptics \\
\hline 5 & Dharshiyani et. al. & 2012 & India & 1 & Mandible & $\begin{array}{l}\text { Antisepsis, larvae removal, } \\
\text { anthelmintic, antibiotics }\end{array}$ \\
\hline 6 & Biradar et al. & 2015 & India & 2 & Buccal mucosa & $\begin{array}{l}\text { Mechanical removal, surgical debridement, } \\
\text { antibiotics }\end{array}$ \\
\hline
\end{tabular}

carcinoma have been reported in the literature (Carvalho et al., 2008; Gabriel et al., 2008; Pessoa and Galvão, 2011; Biradar et al., 2015). Some reports have documented the occurrence of myiasis in association with skin cancer including the head and neck region (Sesterhenn et al., 2009; Wollina et al., 2014). However, the literature is scarce regarding oral myiasis in association with squamous cell carcinoma and none has been written about the subject in English literature. Moreover, to our best knowledge, an approved and effective treatment concept for cases of oral myiasis in malignant wounds patients does not exist. Hence the purpose of the present report was to review cases of oral myiasis associated with oral squamous cell carcinoma, and also to highlight the preventive and treatment concepts in such cases.

\section{Materials and Methods}

A literature search was made in March 2015 using PUBMED for articles relating to the occurrence of myiasis in oral SCC. The search was limited to articles published in English. We used the following search strings: oral myiasis and malignancy; oral myiasis and oral cancer; myiasis and oral squamous cell carcinoma; myiasis and oral cancer. Head and neck SCC and cutaneous metastases were excluded.

\section{Results}

Only six reports (case reports) related to the occurrence of myiasis in oral SCC were identified in the English literature (Table 1)

\section{Discussion}

Myiasis is frequently seen in patients with risk factors such as advanced age, low socioeconomic class, poor hygiene, alcoholics, chronic diseases such as uncontrolled diabetes mellitus and peripheral vascular disease, and mental disorders (Sharma et al., 2008; Atunes et al., 2011). Oral myiasis is a rare entity, and may present as an oral mucosal swelling, gum swelling, periodontal disease, or palatal ulcerations (Biradar et al., 2015). The risk factors for the development of oral myiasis are open wounds, suppurative lesions, and extraction wounds, especially in individuals with neurological deficit (Sankari and Ramakrishnan, 2010; Bhagawati et al., 2013; Aggrawal et al., 2014). In such cases, neglected oral hygiene and halitosis attracts the flies to lay eggs in oral wounds resulting in oral myiasis (Biradar et al., 2015). Myiasis is also a possible complication of advanced and neglected SCC of oral cavity (Gabriel et al., 2008; Biradar et al., 2015). Patients affected by malignant wound or cavity infestations by maggots are characterized by the presence of advanced, giant, and neglected oral SCC (Gabriel et al., 2008; Biradar et al., 2015). Such patients are usually of low social class, malnourished, having poor oral hygiene, and usually live alone and in social isolation. The association of myiasis with oral malignancy can be attributed to the ulcerated and necrotic lesions exposed to the environment resulting from advanced and neglected cancerous lesions (a very common in developing countries). This kind of tissue is attractive to different species of flies, which may deposit their eggs in these medium, especially in the spring and summer months (Sesterhenn et al., 2009; Raposo et al., 2012; Wollina et al., 2014; Biradar et al., 2015). Since proper oral hygiene and hygiene of the surrounding is not maintained by the oral cancer patients as well as the care givers, and other predisposing factors such as the advancing age, alcoholism, diabetes and other systemic conditions exist, the risk for oral Myiasis increases (Biradar et al., 2015).

Extensive myiasis infestations associated with oral SCC have been reported in the literature, though they are very few (Gopalakrishnan et al., 2008; Carvalho et al. 2008; Pessoa L, Galvão 2011; Gabriel et al., 2008; Biradar et al., 2015). It is possible that this condition is underreported, as oral cancer is a common malignancy. In this literature review, we identified only six published articles (case reports) that reported the occurrence of myiasis in association with oral squamous cell carcinoma (Table1). Carvalho et al. reported a case of myiasis in a patient with squamous cell carcinoma without physical or neurological deficiency. Gopalakrishnan et al. have reported 2 cases of myiasis associated with oral SCC: one case was on the buccal mucosa and the other was on lower lip. The authors also described 2 more myiasis cases associated with malignancy on the neck. Pessoa and Galvão reported one case of myiasis in advanced squamous cell carcinoma of the mandible. The affected patient was a 44-year old man, dependent on alcohol and tobacco, living in a rural area with low economic status and having poor living conditions. In Brazil, Gabriel et al., reported one case of extensive orofacial myiasis associated with SCC of the floor of the mouth. The reported patient was a 72-year old woman, living in a rural 
area. Dharshiyani et al. reported a case of oral myiasis associated with SSC on the mandible. The patient was 75-year old male, chronic alcoholic and belonged to a very low socioeconomic status. Biradar et al. reported two cases of oral myiasis associated with oral SCC in adult males. In the both reported cases, the cancerous ulcers started on the buccal mucosa, which progressed to involve the whole adjacent tissues, with marked extra-oral ulceration.

From the above reported cases, it is evident that all patients are of low socioeconomic status having poor living conditions, and most of them lived in rural areas with evidence of social and family alienation. This situation can be observed by the clinically advanced stage of the malignancy and its long-term, indicating negligence and lack of knowledge.

Although there is no specific treatment modality for the myiasis, the simplest is the mechanical removal of the larvae with tweezers, usually under local anesthesia. However, mechanical removal is not sufficient treatment because of the larva's hooks that it uses to grip the tissue cavity (Verma, Marya, 2011; Dharshiyani et al., 2012). Application of ether to the wound has been suggested in order to compel the maggots wiggle out of the host tissue. The wounds can be debrided by irrigating with normal saline or antiseptic medicaments such as $0.2 \%$ aqueous chlorhexidine, iodoform, ethyl chloride, mercuric chloride, creosote or turpentine oil. Whitehead varnish pack, which contains ether, can be applied to the raw wound for protection during the healing phase 3 (Dharshiyani et al., 2012). Recently, Ivermectin, a semisynthetic macrolide antibiotic used in veterinary medicine, has been applied to the treatment of oral myiasis. This medication activates the release of $\gamma$-aminobutyric acid which induces the death of the larvae and their spontaneous elimination (Biradar et al., 2015).

In conclusion, myiasis is a possible association of advanced and ulcerated oral SCC that needs attention. Poor living and hygienic conditions, mainly among the homeless, aged, or debilitating persons may predispose to cases of myiasis, mainly in the tropical countries. Prevention of myiasis can be achieved by controlling fly population, maintaining good oral and general cleanliness such as cleaning and dressing the cancerous wounds and by educating the susceptible population where sanitation is meager. Furthermore, education of the suspetiple populations in developing countries about such diseases is clearly indicated..

\section{References}

Aggarwal A, Daniel MJ, Shetty RS, et al (2014). Oral Myiasis Caused by Chrysomya bezziana in Anterior Maxilla. Case Rep Dent, 2014, 518427.

Antunes AA, Santos Tde S, Avelar RL, et al (2011). Oral and maxillofacial myiasis: a case series and literature review. Oral Surg Oral Med Oral Pathol Oral Radiol Endod, 112, 81-5.

Bhagawati B, Gupta M, Singh S (2013). Oral myiasis: A rare entity. European Journal of General Dentistry, $2,312$.

Biradar S, Wankhede P, Munde A, et al (2015). Extensive Myiasis infestation associated with Oral Squamous Cell Carcinoma: Report of two cases. Dent Res J (Isfahan), 12, 100-5.
Carvalho RW, Santos TS, Antunes AA, et al (2008). Oral and maxillofacial myiasis associated with epidermoid carcinoma: a case report. J Oral Sci, 50, 103-5.

Dharshiyani SC, Wanjari SP, Wanjari PV, et al (2012). Oral squamous cell carcinoma associated with myiasis. $B M J$ Case Rep, 2012.

Ferlay J, Shin HR, Bray F, et al (2010). Estimates of worldwide burden of cancer in 2008: GLOBOCAN 2008. Int J Cancer, 127, 2893-917.

Gabriel JG, Marinho SA, Verli FD, et al (2008). Extensive myiasis infestation over a squamous cell carcinoma in the face. Case report. Med Oral Patol Oral Cir Bucal, 13, 9-11.

Gopalakrishnan S, Srinivasan R, Saxena SK, et al (2008). Myiasis in different types of carcinoma cases in southern India. Indian J Med Microbiol, 26, 189-92.

Krishna Rao SV, Mejia G, Roberts-Thomson K, et al (2013). Epidemiology of oral cancer in Asia in the past decade--an update (2000-2012). Asian Pac J Cancer Prev, 14, 5567-77.

Laurence SM, "Dipterous larvae infection," British Medical Journal, 9, 1909.

Pereira T, Tamgadge AP, Chande MS, et al (2010). Oral myiasis. Contemp Clin Dent, 1, 275-6.

Pessoa L, Galvao V (2011). Myiasis infestation in advanced oral squamous cell carcinoma. BMJ Case Rep, 2011.

Raposo AA, Schettini AP, Massone C (2012). Concurrent primary and secondary myiasis on basal cell carcinoma. $A n$ Bras Dermatol, 87, 292-5.

Sankari LS, Ramakrishnan K (2010). Oral myiasis caused by Chrysomya bezziana. J Oral Maxillofac Pathol, 14, 16-8.

Saravanan T, Mohan MA, Thinakaran M, et al (2015). Oral myiasis. Indian J Palliat Care, 21, 92-4.

Sesterhenn AM, Pfutzner W, Braulke DM, et al (2009). Cutaneous manifestation of myiasis in malignant wounds of the head and neck. Eur J Dermatol, 19, 64-8.

Sharma J, Mamatha GP, Acharya R (2008). Primary oral myiasis: a case report. Med Oral Patol Oral Cir Bucal, 13, 714-6.

Verma N, Marya J (2011). Oral myiasis in maxillofacial traumatreated with Ivermectin. Int J Dental Clinics, 3.

Wollina U (2015). Myiasis on squamous cell carcinoma of skin. Wien Med Wochenschr, 165, 79-82.

Zumpt F. Myiasis in man and animals in the old world. In: Zumpt F, editor (1965). A Textbook for Physicians, Veterinarians and Zoologists. London: Butterworth and Co. Ltd; p.109. 